\title{
Bacterial Community Profile of the Gut Microbiota Differs between Hypercholesterolemic Subjects and Controls
}

\author{
Camilo Rebolledo, ${ }^{1}$ Alejandro Cuevas, ${ }^{2}$ Tomás Zambrano, ${ }^{1}$ \\ Jacquelinne J. Acuña, ${ }^{3}$ Milko A. Jorquera, ${ }^{3}$ Kathleen Saavedra, ${ }^{1}$ Claudia Martínez, ${ }^{4}$ \\ Fernando Lanas, ${ }^{1}$ Pamela Serón, ${ }^{1}$ Luis A. Salazar, ${ }^{1}$ and Nicolás Saavedra ${ }^{1}$ \\ ${ }^{1}$ Center of Molecular Biology and Pharmacogenetics, Department of Basic Sciences, Faculty of Medicine, \\ Universidad de La Frontera, Temuco, Chile \\ ${ }^{2}$ Department of Preclinical Sciences, Faculty of Medicine, Universidad de La Frontera, Temuco, Chile \\ ${ }^{3}$ Applied Microbial Ecology Laboratory, Department of Chemical Sciences and Natural Resources, \\ Scientific and Technological Bioresource Nucleus, Universidad de La Frontera, Temuco, Chile \\ ${ }^{4}$ Faculty of Health, Universidad Santo Tomás, Temuco, Chile \\ Correspondence should be addressed to Nicolás Saavedra; nicolas.saavedra@ufrontera.cl
}

Received 13 March 2017; Revised 18 May 2017; Accepted 23 May 2017; Published 15 June 2017

Academic Editor: Clara G. de los Reyes-Gavilan

Copyright (C) 2017 Camilo Rebolledo et al. This is an open access article distributed under the Creative Commons Attribution License, which permits unrestricted use, distribution, and reproduction in any medium, provided the original work is properly cited.

\begin{abstract}
The role of gut microbiota in the development of metabolic illnesses has been abundantly demonstrated. Recent studies suggest that gut microbiota alterations may also be related to the development of hypercholesterolemia. Therefore, we aimed to assess differences in the gut bacterial community profiles between hypercholesterolemic subjects and controls. Thirty cases diagnosed with hypercholesterolemia and 27 normocholesterolemic controls were included. A fasting whole blood sample was obtained to determine the lipid profile. In parallel, stool samples were collected and total DNA was isolated to assess the bacterial community profiles by denaturing gradient gel electrophoresis (DGGE). In addition, the Richness, Shannon-Weaver, and Simpson indexes were used to evaluate the richness and diversity of bacterial communities. As expected, serum concentrations of total cholesterol, triglycerides, and LDL-cholesterol were significantly higher in the cases compared with controls. Moreover, DGGE analysis showed a lower richness and diversity of bacterial communities in hypercholesterolemic subjects. In conclusion, our results showed differences in the profiles of bacterial communities between hypercholesterolemic subjects and controls, suggesting a possible role of the gut microbiota in the development of hypercholesterolemia.
\end{abstract}

\section{Introduction}

Cardiovascular diseases (CVD) are the leading cause of death in the world, causing annually about 16 million deaths and more than 21,000 in Chile $[1,2]$. Projections are not promising, since it is expected that by 2030 these numbers should increase [3]. Among CVD, cardiac and cerebral ischemic diseases correspond to the most frequent presentations, having as a common etiologic mechanism the development of atherosclerosis in arteries irrigating the compromised tissues [4]. On the other hand, dyslipidemias are considered among the most important risk factors contributing to CVD development. Considering that hypercholesterolemia, defined by the elevation of circulating low-density lipoprotein levels, increases the risk of coronary artery disease, its treatment-besides reducing cholesterol levels-has been shown to decrease both incidence and mortality by acute coronary events $[5,6]$.

During the last years, gut microbiota has been related to both the maintenance of health and the development of several diseases [7]. The role of microbiota in disease development has been strongly demonstrated in the case of inflammatory bowel diseases (IBD) such as Crohn's disease and ulcerative colitis $[8,9]$. In this context, changes in the bacterial profiles have been also associated with metabolic disturbances such as obesity and type 2 diabetes 
mellitus $[10,11]$. Recent studies have suggested the influence of changes in the microbiota on lipid metabolism in animal models, describing differences in both cholesterol absorption and serum levels between germ-free and conventional rats $[12,13]$. Moreover, studies performed in hamsters-animals showing human-like lipoprotein metabolism [14]—have shown that specific bacterial groups found in stool samples are related to improvements in lipid metabolism induced by diet changes [15].

Nowadays, precision medicine has sought to improve the understanding of the health-disease process, where a broader description of factors involved in the development of a particular pathology becomes highly relevant. The prior statement led to suggesting that gut microbiota characterization represents a useful aspect into a better comprehension of the cardiovascular health status of an individual [16]. Considering these antecedents, the aim of the present study was to assess differences in the gut bacterial community profiles between hypercholesterolemic subjects and controls.

\section{Materials and Methods}

2.1. Subjects. We design a case-control study including 30 hypercholesterolemic subjects (cases) diagnosed according to the NCEP criteria [17] and 27 normocholesterolemic subjects (controls), selected at the Center for Cardiovascular and Internal Medicine Studies, Universidad de La Frontera (Temuco, Chile). Anthropometric parameters such as systolic blood pressure (SBP) and diastolic blood pressure (DBP), weight, and height were measured. Individuals diagnosed with IBD, diabetes mellitus, and obesity were excluded considering that these pathologies have been associated with changes in the composition of the gut microbiota. Individuals with history of antibiotic therapy during the prior 6 months were also excluded. The present study was conducted based on the Declaration of Helsinki, and all individuals agreed voluntarily to participate by signing a written informed consent, previously approved by the Scientific Ethics Committee of Universidad de La Frontera (number 030/2015).

2.2. Biochemical Determinations. Biochemical measurements were performed on serum samples obtained from total blood using standard venipuncture techniques following a 12-h overnight fast. Glucose, triglycerides (TG), total cholesterol (TC), and high-density lipoprotein cholesterol (HDL-C) were quantified using enzymatic-colorimetric methods. Low-density lipoprotein cholesterol (LDL-C) was calculated using Friedewald's formula when TG did not exceed $4.52 \mathrm{mmol} / \mathrm{L}$. Quality of the determinations was controlled by using normal and pathological commercial serums (Human, Germany).

2.3. Molecular Analysis. Stool samples were collected to analyze bacterial community profiles of the gut microbiota by gel electrophoresis with denaturing gradient (DGGE) technique. For the DGGE, DNA was isolated from deposition samples using the Fast DNA Spin (MP Biomedicals, USA) commercial kit, following the manufacturer's instructions. DNA was later quantified by UV spectrophotometry with Infinite ${ }^{\circledR} 200 \mathrm{PRO}$ Nanoquant microplate reader (Tecan, Switzerland). Then, $100 \mathrm{ng}$ DNA was used to amplify a $454 \mathrm{bp}$ fragment of the $16 \mathrm{~S}$ rRNA gene by touchdown PCR using the EUBf933-GC and EUBr1387 primers [18].

PCR was performed in $50 \mu \mathrm{L}$ of final volume containing $\mathrm{MgCl}_{2}(3 \mathrm{mM})$, GoTaq ${ }^{\circledR}$ Flexi Buffer $(1 \mathrm{x})$, dNTPs (0.2 mM each), GoTaq FlexiDNA polymerase (1.25 units), and primers (200 nM each). Touchdown PCR was performed on a MultiGene ${ }^{\mathrm{TM}}$ thermocycler (Labnet International, Inc., USA) using the following cycling conditions: denaturation at $94^{\circ} \mathrm{C}$ for 1 minute, followed by a hybridization step using a $0.5^{\circ} \mathrm{C}$ decrease for each cycle between 65 and $55^{\circ} \mathrm{C}$, with 10 additional cycles at $55^{\circ} \mathrm{C}$, and the extension step at $72^{\circ} \mathrm{C}$ for 3 minutes. A final extension of 7 minutes was included. PCR products were visualized by electrophoresis on a $2 \%$ agarose gel using a 100 bp DNA marker stained with GelRed ${ }^{\mathrm{TM}}$ (Biotium, USA).

Subsequently, PCR products were loaded onto a DGGE gel (acrylamide/bisacrylamide in 37.5:1 ratio) at 8\% (w/v) in $1 \mathrm{x}$ TAE buffer $(20 \mathrm{mM}$ acetic acid and $1 \mathrm{mM}$ EDTA $\mathrm{pH}$ 8.0). A gradient of 40 to $60 \%$ of denaturing agents (urea $7 \mathrm{M}$ and $40 \%$ formamide) was used in DGGE gel preparation. Electrophoretic separation was performed at $20 \mathrm{~V}$ for the initial 10 minutes and then at $80 \mathrm{~V}$ for 17 hours at constant $60^{\circ} \mathrm{C}$. Finally, DGGE gels were stained using SYBR ${ }^{\circledR}$ Gold $\left(\right.$ Invitrogen ${ }^{\mathrm{TM}}$ ) and the images were acquired using a GeldocIt $^{\circledR}$ TS2 310 photodocumentation system (UVP, USA). Banding patterns were analyzed in the CLIQs 1D Pro software (Totallab Ltd., UK). A nonmetric multidimensional scaling (nMDS) data matrix incorporating the Bray-Curtis similarity index was obtained from the Primer6 software (Primer-E Ltd., UK). In addition, we analyzed the pattern of bands to evaluate richness and diversity by the Shannon-Weaver and Simpson indexes.

2.4. Statistical Analysis. Statistical analysis was performed using the IBM SPSS Statics software for Mac, version 23.0 (IBM Corp., USA). Data are presented as averages \pm standard deviation (SD). Differences between means of continuous variables were evaluated by Student's $t$-test. Statistical significance was set at $p<0.05$.

\section{Results}

Anthropometric and biochemical variables of the subjects are summarized in Table 1. Analyzed individuals were aged between 40 and 80 years, with no significant differences between the two groups. The results did not show significant differences for SBP and DBP parameters. As expected, serum concentrations of TC and LDL-C were significantly higher in cases $(6.13$ and $3.99 \mathrm{mmol} / \mathrm{L}$, resp.) compared with controls (5.36 and $3.01 \mathrm{mmol} / \mathrm{L}$, resp.). These individuals also showed higher elevated glucose and TG levels (5.70 and $1.91 \mathrm{mmol} / \mathrm{L}$, resp.) compared with controls (5.22 and $1.18 \mathrm{mmol} / \mathrm{L}$, resp.). However, no significant differences were identified for HDL-C levels. Cases also showed significantly higher BMI $\left(24 \mathrm{Kg} / \mathrm{m}^{2}\right)$ than controls $\left(23 \mathrm{Kg} / \mathrm{m}^{2}\right)$. 
TABLE 1: Clinical and demographic characteristics of hypercholesterolemic and controls subjects.

\begin{tabular}{lccc}
\hline Parameter & Cases & Controls & $p$ value \\
\hline Age, years & $64.0 \pm 1.9$ & $60.1 \pm 2.2$ & 0.100 \\
SBP, $\mathrm{mm} \mathrm{Hg}$ & $127.1 \pm 3.3$ & $122.0 \pm 4.1$ & 0.328 \\
DBP, $\mathrm{mm} \mathrm{Hg}$ & $81.2 \pm 1.7$ & $80.0 \pm 2.3$ & 0.655 \\
BMI, Kg/m & $24.2 \pm 0.4$ & $22.9 \pm 0.4$ & 0.023 \\
Glucose, $\mathrm{mmol} / \mathrm{L}$ & $5.70 \pm 0.13$ & $5.22 \pm 0.12$ & 0.008 \\
TC, $\mathrm{mmol} / \mathrm{L}$ & $6.13 \pm 0.30$ & $5.36 \pm 0.16$ & 0.029 \\
LDL-C, $\mathrm{mmol} / \mathrm{L}$ & $3.99 \pm 0.27$ & $3.01 \pm 0.12$ & 0.002 \\
HDL-C, $\mathrm{mmol} / \mathrm{L}$ & $1.62 \pm 0.09$ & $1.60 \pm 0.06$ & 0.840 \\
TG, $\mathrm{mmol} / \mathrm{L}$ & $1.91 \pm 1.18$ & $1.18 \pm 0.09$ & 0.001 \\
\hline
\end{tabular}

SBP: systolic blood pressure; DBP: diastolic blood pressure; BMI: body mass index; TC: total cholesterol; LDL-C: low-density lipoprotein cholesterol; HDL-C: high-density lipoprotein cholesterol; TG: triglycerides.

TABle 2: Shannon-Weaver, Simpson, and Richness indexes of bacterial community profiles from hypercholesterolemic subjects and controls.

\begin{tabular}{lccc}
\hline Index & Cases & Controls & $p$ value \\
\hline Shannon-Weaver & $1.80 \pm 0.43$ & $2.07 \pm 0.43$ & 0.019 \\
Simpson & $6.23 \pm 2,92$ & $7.02 \pm 2.62$ & 0.025 \\
Richness & $7.10 \pm 3.24$ & $9.41 \pm 3.15$ & 0.009 \\
\hline
\end{tabular}

With respect to the molecular analysis performed by DGGE, we observed that the banding profiles differ between cases and controls, which in general were grouped in the two main branches in the dendrogram (Figure 1).

Then, this difference was also confirmed by plotting the nMDS analysis, which shows that cases are similar to each other, forming a well-defined grouping (Figure 2).

Finally, we found significant differences for the Richness, Shannon-Weaver, and Simpson indexes of the banding profiles between both groups, with a noticeable decrease of the three indexes used in cases with respect to the controls (Table 2).

\section{Discussion}

A very well established association exists between hypercholesterolemia and CVD risk. However, up until now, the wide spectrum of CVD determinants is yet far to be fully understood. Very recently, a clear association between variations in gut microbiota and disease development has been demonstrated, showing a growing interest in the relationship between gut microbes and lipid profile. In the present study, we explored possible differences between bacterial community profiles of the gut tract of hypercholesterolemic individuals and controls. By calculating diversity indexes obtained from the analysis of bacterial DNA from the gut, we have consistently demonstrated that hypercholesterolemic subjects have significantly lower bacterial diversity compared to control individuals. In addition, the nMDS scaling analysis demonstrated the existence of differences among cases and controls, allowing the grouping by similarity of 26 from the 29 hypercholesterolemic individuals.
Data obtained are concordant with a 2015 cross-sectional study relating microbiota with BMI, TG, and HDL-C, revealing that healthy lipid levels were associated with an increase in microbial diversity [19]. Moreover, a recent and highly relevant review provided insight into the relationship between the gut microbiota and interindividual variation in the lipid profile, suggesting that gut microbes can explain a significant part of these differences [20]. The calculated indexes, which account for bacterial diversity, reflected that significant differences exist in the number of phylotypes observed, also showing differences in the relative abundance of a taxonomic unit between the studied groups.

An interesting result of our study is that hypercholesterolemic individuals presented lower richness and lower diversity compared with control individuals. It has been suggested that attenuation in phylotypes number and alterations in bacterial relative abundance as showed by the studied hypercholesterolemic subjects could modulate cholesterol absorption or modulate cholesterol metabolism at the systemic level. In line with this observation, it has been demonstrated that facultative and anaerobic bacteria located in the large bowel produce secondary bile acids from bile salts secreted by the individual, which can modulate lipid metabolism at both hepatic and systemic levels [21]. On the other hand, the attenuation of certain communities of gut microorganisms could determine a lower production of short chain fatty acids (SCFAs), which have been shown to reduce plasma concentrations of cholesterol in rodents and humans [22], or they could mediate L-carnitine metabolism into forming trimethylamine $\mathrm{N}$-oxide, a compound involved in the reverse transport of cholesterol, cholesterol and sterols metabolism, and the modulation of bile acids amount and composition [23].

The approach used in the present study does not permit to identify the specific communities that differ between the groups. However, since it has been suggested that differences in microbial diversity could directly affect hepatic metabolism by exerting an influence on hepatic lipid biosynthesis [24] as well as lipids degradation [25] due to its proximity to the gastrointestinal tract, differences described in the present study might affect this mechanism depending on the bacterial communities differing between 


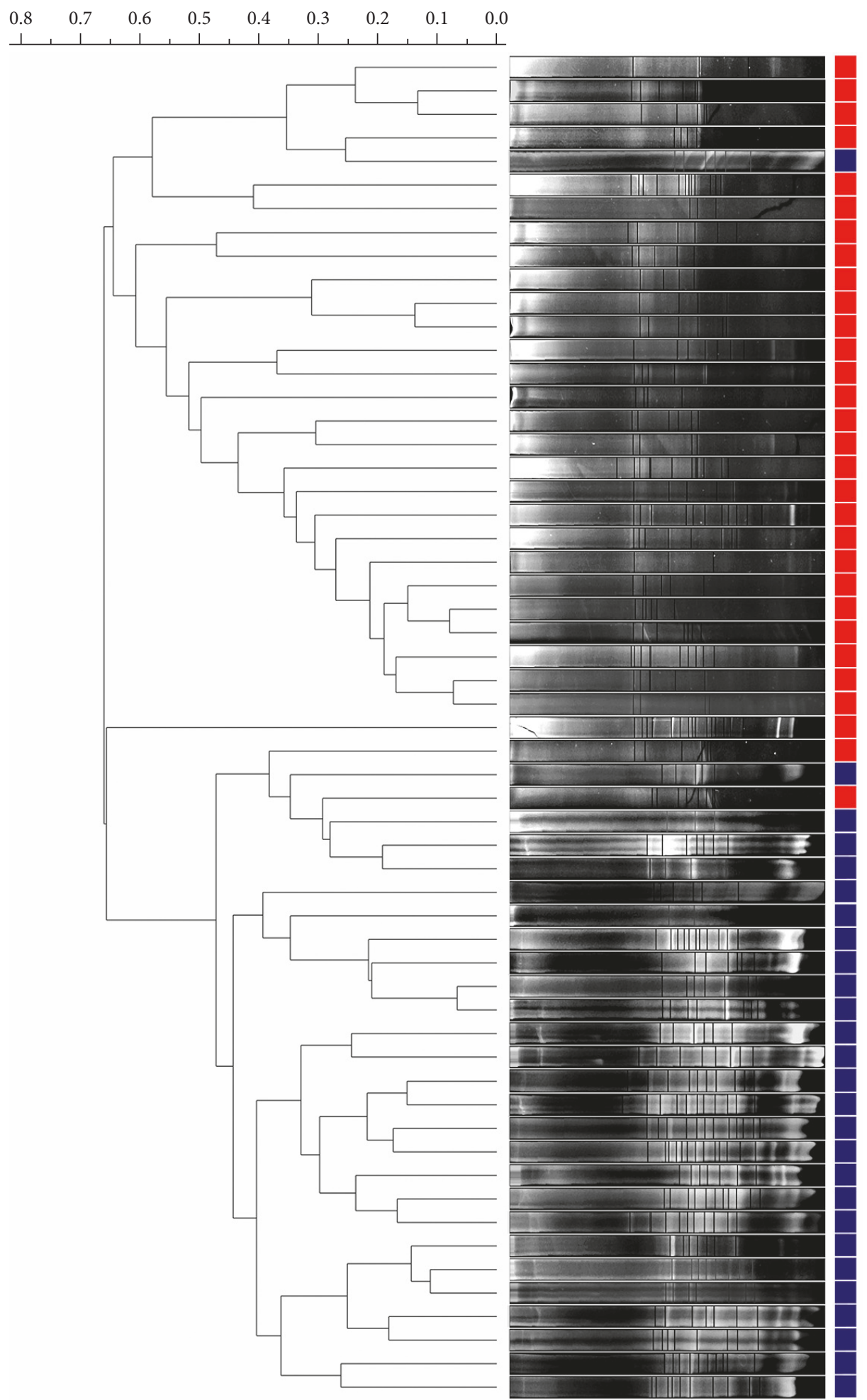

FIGURE 1: Dendrogram of bacterial community profiles in stool samples from hypercholesterolemic subjects (red) and controls (blue).

hypercholesterolemic and control individuals. In this line, Claus et al. demonstrated that gut microbiota stimulates an increase in hepatic triglyceride synthesis and modulates essential lipid absorption regulators, such as taurocholate and tauromuricholate, associating this effect to the Coriobacteriaceae family [26]. Gut microbiota is also able to modify lipid fractions in serum, adipose tissue, and liver [27]. For example, Erysipelotrichi (Firmicutes phylum) has been 


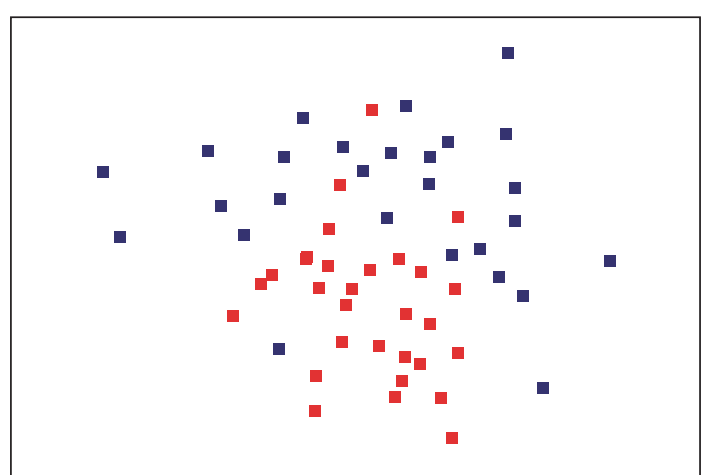

FIGURE 2: nMDS analysis of bacterial community profiles in stool samples from hypercholesterolemic subjects (red) and controls (blue).

associated with both lipid impairment in animal models of hypercholesterolemia and with the development of fatty liver in humans $[28,29]$.

Although the microbiota in hypercholesterolemic individuals has not been characterized, a possible role of bacteria as lipid profile modulators in humans has been reported in individuals whose diet was supplemented with probiotics, which are defined as live microorganisms administered in suitable amounts to confer health benefits on the host. Two randomized, double-blind, placebo-controlled studies showed a $4.4 \%$ TC and 6.2\% LDL-C reduction caused by dairy products enriched with Lactobacillus acidophilus or Enterococcus faecium, in subjects having a normal lipid profile and subjects with medium to moderate hypercholesterolemia $[30,31]$. In addition, the lipid-lowering effect of Lactobacillus reuteri was described as an effect associated with increased intraluminal bile acid deconjugation, resulting in reduced absorption of sterols different than cholesterol [32].

Among the limitations of our study, we need to mention that DGGE allows describing qualitatively the bacterial populations present in a particular sample. However, as aforementioned, our approach does not permit to identify which microorganisms could be differentially contained in the group of hypercholesterolemic subjects. Despite this, from a more general perspective, the nMDS ordination plot of the DGGE data showed a clear grouping of the microbiota from hypercholesterolemic individuals, which is not so evident in the control group, with the exception of some of these individuals that appear overlapping with the control group, suggesting that there is a group of predominant phylotypes influencing hypercholesterolemia.

Finally, the technical limitations could be corrected in future studies by analyzing microorganisms by means of next-generation sequencing (NGS) methodologies, allowing a much deeper description of the bacterial populations associated with the development of hypercholesterolemia.

\section{Conclusion}

Our results showed differences in the profiles and diversity of bacterial communities between hypercholesterolemic subjects and controls, suggesting a possible role of the gut microbiota in the development of hypercholesterolemia.

\section{Conflicts of Interest}

The authors declare that there are no conflicts of interest regarding the publication of this article.

\section{Acknowledgments}

This study was supported by grants from Dirección de Investigación of Universidad de La Frontera (DIUFRO) through Projects of Associative Investigation (PIA15-0001) and by FONDECYT-Chile (Grant no. 11160364). Milko A. Jorquera thanks the Project CONICYT-USA (USA2013-0010).

\section{References}

[1] WHO, World Health Organization, Data and statistics 2013, http://www.who.int/research/es/.

[2] DEIS, "Departamento de Estadísticas e Información en Salud, Ministerio de Salud, Chile, 2013".

[3] C. D. Mathers and D. Loncar, "Projections of global mortality and burden of disease from 2002 to 2030," PLoS Medicine, vol. 3, no. 11, article e442, 2006.

[4] E. P. Soler and V. C. Ruiz, "Epidemiology and risk factors of cerebral ischemia and ischemic heart diseases: similarities and differences," Current Cardiology Reviews, vol. 6, no. 3, pp. 138149,2010

[5] P. S. Yusuf, S. Hawken, S. Ônpuu et al., "Effect of potentially modifiable risk factors associated with myocardial infarction in 52 countries (the INTERHEART study): case-control study," The Lancet, vol. 364, no. 9438, pp. 937-952, 2004.

[6] C. Baigent, A. Keech, P. M. Kearney, L. Blackwell, G. Buck, C. Pollicino et al., "Efficacy and safety of cholesterol-lowering treatment: prospective meta-analysis of data from 90,056 participants in 14 randomised trials of statins," The Lancet, vol. 366, no. 9493, pp. 1267-1278, 2005.

[7] A. B. Shreiner, J. Y. Kao, and V. B. Young, "The gut microbiome in health and in disease," Current Opinion in Gastroenterology, vol. 31, no. 1, pp. 69-75, 2015.

[8] L. Jostins, S. Ripke, R. K. Weersma et al., "Host-microbe interactions have shaped the genetic architecture of inflammatory bowel disease," Nature, vol. 491, no. 7422, pp. 119-124, 2012.

[9] D. Knights, K. G. Lassen, and R. J. Xavier, "Advances in inflammatory bowel disease pathogenesis: linking host genetics and the microbiome," Gut, vol. 62, no. 10, pp. 1505-1510, 2013.

[10] A. Everard and P. D. Cani, "Diabetes, obesity and gut microbiota," Best Practice and Research: Clinical Gastroenterology, vol. 27, no. 1, pp. 73-83, 2013.

[11] A. V. Hartstra, K. E. C. Bouter, F. Bäckhed, and M. Nieuwdorp, "Insights into the role of the microbiome in obesity and type 2 diabetes," Diabetes Care, vol. 38, no. 1, pp. 159-165, 2015.

[12] H. Danielsson and B. Gustafsson, "On serum-cholesterol levels and neutral fecal sterols in germ-free rats. Bile acids and steroids 59," Archives of Biochemistry and Biophysics, vol. 83, no. 2, pp. 482-485, 1959.

[13] B. S. Wostmann, "Intestinal bile acids and cholesterol absorption in the germfree rat," The Journal of Nutrition, vol. 103, no. 7, pp. 982-990, 1973. 
[14] P. L. Mitchell and R. S. McLeod, "Conjugated linoleic acid and atherosclerosis: studies in animal models," Biochemistry and Cell Biology, vol. 86, no. 4, pp. 293-301, 2008.

[15] I. Martínez, G. Wallace, C. Zhang et al., "Diet-induced metabolic improvements in a hamster model of hypercholesterolemia are strongly linked to alterations of the gut microbiota," Applied and Environmental Microbiology, vol. 75, no. 12, pp. 4175-4184, 2009.

[16] E. M. Antman and J. Loscalzo, "Precision medicine in cardiology," Nature Reviews Cardiology, vol. 13, no. 10, pp. 591-602, 2016.

[17] S. M. Grundy, J. I. Cleeman, C. N. Bairey Merz et al., "Implications of recent clinical trials for the National Cholesterol Education Program Adult Treatment Panel III guidelines," Circulation, vol. 110, no. 2, pp. 227-239, 2004.

[18] T. Iwamoto, K. Tani, K. Nakamura et al., "Monitoring impact of in situ biostimulation treatment on groundwater bacterial community by DGGE," FEMS Microbiology Ecology, vol. 32, no. 2, pp. 129-141, 2000.

[19] J. Fu, M. J. Bonder, M. C. Cenit et al., “The gut microbiome contributes to a substantial proportion of the variation in blood lipids," Circulation Research, vol. 117, no. 9, pp. 817-824, 2015.

[20] Z. Wang, D. Koonen, M. Hofker, and J. Fu, "Gut microbiome and lipid metabolism: From associations to mechanisms," Current Opinion in Lipidology, vol. 27, no. 3, pp. 216-224, 2016.

[21] J. M. Ridlon, D. J. Kang, P. B. Hylemon, and J. S. Bajaj, "Bile acids and the gut microbiome," Current Opinion in Gastroenterology, vol. 30, no. 3, pp. 332-338, 2014.

[22] T. Fushimi, K. Suruga, Y. Oshima, M. Fukiharu, Y. Tsukamoto, and T. Goda, "Dietary acetic acid reduces serum cholesterol and triacylglycerols in rats fed a cholesterol-rich diet," British Journal of Nutrition, vol. 95, no. 5, pp. 916-924, 2006.

[23] R. A. Koeth, Z. Wang, B. S. Levison et al., "Intestinal microbiota metabolism of L-carnitine, a nutrient in red meat, promotes atherosclerosis," Nature Medicine, vol. 19, no. 5, pp. 576-585, 2013.

[24] F. Bäckhed, H. Ding, T. Wang et al., "The gut microbiota as an environmental factor that regulates fat storage," Proceedings of the National Academy of Sciences of the United States of America, vol. 101, no. 44, pp. 15718-15723, 2004.

[25] F. Bäckhed, J. K. Manchester, C. F. Semenkovich, and J. I. Gordon, "Mechanisms underlying the resistance to diet-induced obesity in germ-free mice," Proceedings of the National Academy of Sciences of the United States of America, vol. 104, no. 3, pp. 979-984, 2007.

[26] S. P. Claus, S. L. Ellero, B. Berger et al., "Colonization-induced host-gut microbial metabolic interaction," mBio, vol. 2, no. 2, 2011.

[27] V. R. Velagapudi, R. Hezaveh, C. S. Reigstad et al., "The gut microbiota modulates host energy and lipid metabolism in mice," Journal of Lipid Research, vol. 51, no. 5, pp. 1101-1112, 2010.

[28] M. D. Spencer, T. J. Hamp, R. W. Reid, L. M. Fischer, S. H. Zeisel, and A. A. Fodor, "Association between composition of the human gastrointestinal microbiome and development of fatty liver with choline deficiency," Gastroenterology, vol. 140, no. 3, pp. 976-986, 2011.

[29] C. Zhang, M. Zhang, S. Wang et al., "Interactions between gut microbiota, host genetics and diet relevant to development of metabolic syndromes in mice," ISME Journal, vol. 4, no. 2, pp. 232-241, 2010.
[30] G. Schaafsma, W. J. A. Meuling, W. Van Dokkum, and C. Bouley, "Effects of a milk product, fermented by Lactobacillus acidophilus and with fructo-oligosaccharides added, on blood lipids in male volunteers," European Journal of Clinical Nutrition, vol. 52, no. 6, pp. 436-440, 1998.

[31] M. C. Bertolami, A. A. Faludi, and M. Batlouni, "Evaluation of the effects of a new fermented milk product (Gaio) on primary hypercholesterolemia," European Journal of Clinical Nutrition, vol. 53, no. 2, pp. 97-101, 1999.

[32] M. L. Jones, C. J. Martoni, and S. Prakash, "Cholesterol lowering and inhibition of sterol absorption by Lactobacillus reuteri NCIMB 30242: A randomized controlled trial," European Journal of Clinical Nutrition, vol. 66, no. 11, pp. 1234-1241, 2012. 

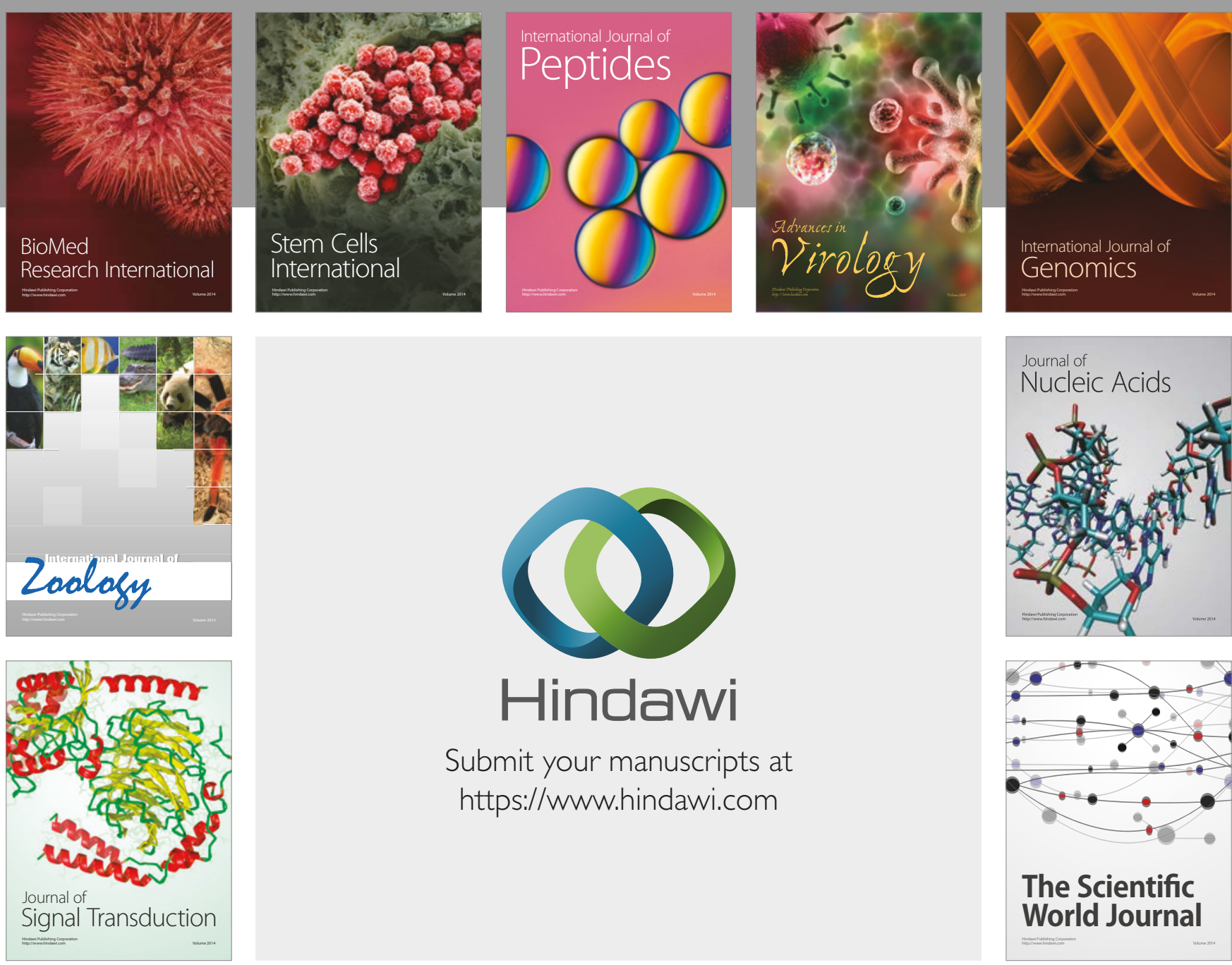

Submit your manuscripts at

https://www.hindawi.com
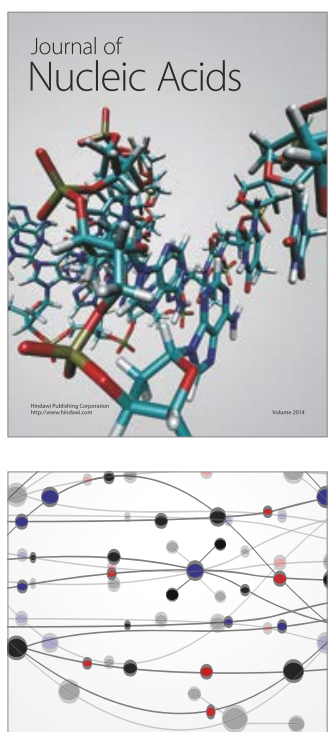

The Scientific World Journal

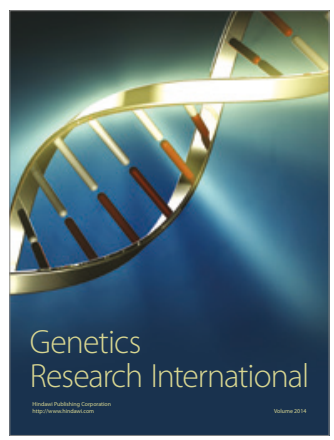

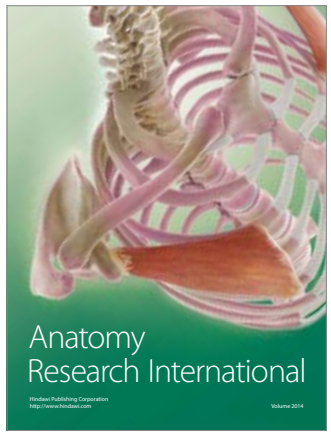

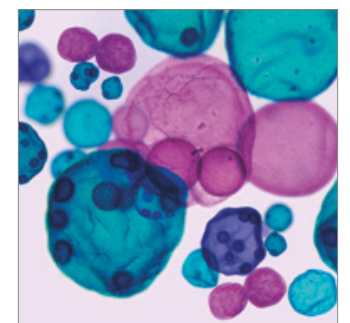

International Journal of Microbiology
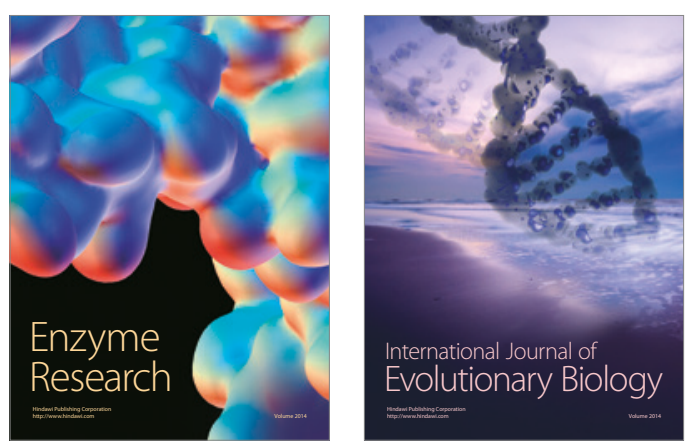
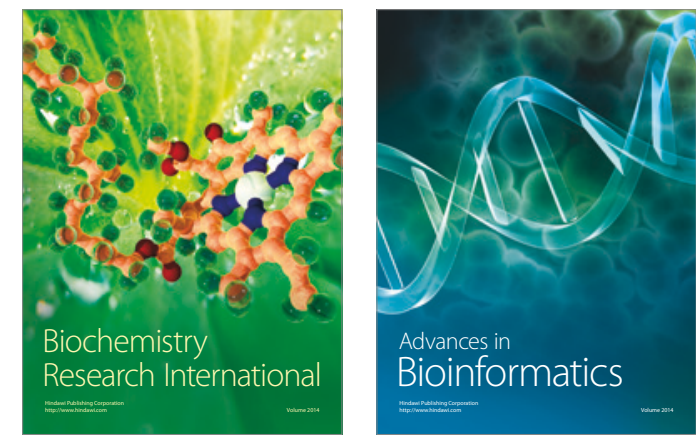

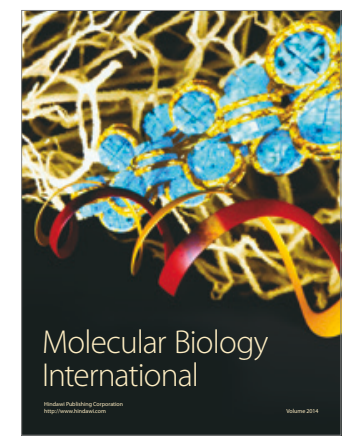

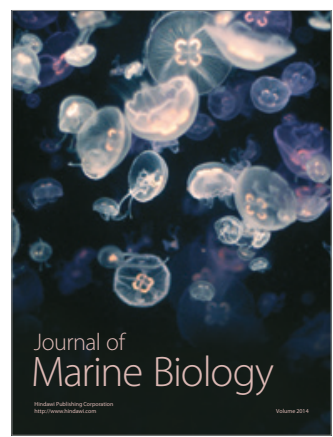

